\title{
Evaluation of Soybean Oil Quality and Establishment of a Predictive Model to Identify Suitable Soybean Varieties for Oil Processing
}

\author{
Lu Sun ${ }^{1}$, Fang Wang ${ }^{1}$, Zheng Xu ${ }^{1}$, Zebin Weng ${ }^{1}$, Haizhao Song ${ }^{1}$, Yong Fang ${ }^{1}$, Xiaozhi \\ Tang $^{1}$, Tuanjie Zhao ${ }^{1}$, and Xinchun SHEN $^{2}$ \\ ${ }^{1}$ Affiliation not available \\ ${ }^{2}$ Nanjing University of Finance and Economics - Xianlin Campus
}

May 5, 2020

\begin{abstract}
Various characteristics of soybeans play important roles in the soybean oil quality. In this study, soybean chemical traits and ten characteristic parameters of soybean oil quality were evaluated for 58 varieties to determine the relationship between soybean chemical traits and the soybean oil quality characteristics by correlation analysis. After 7 of 58 soybean oil variety products were set as outliers by the Box-plot method, an evaluation model for the overall soybean oil quality was established based on ten parameters. Cluster analysis was used to classify 51 soybean oil varieties into three quality grades based on the oil quality evaluation model. A prediction model for the quality of the soybean oil based on the chemical traits of soybeans was constructed by stepwise regression. The relative errors between the actual values and the predicted values of the validation varieties were less than $10 \%$. This study suggested that the total oil, daidzin, total phenolic, stearic acid, linolenic acid and a-tocopherol contents may be useful for predicting the quality of soybean oil. Therefore, our study provides a valuable method to evaluate the quality of soybean oil products and important information for generating soybean varieties for soybean oil processing.
\end{abstract}

\section{Hosted file}

manuscript JAOCS final.doc available at https://authorea.com/users/303149/articles/433274evaluation-of-soybean-oil-quality-and-establishment-of-a-predictive-model-to-identifysuitable-soybean-varieties-for-oil-processing 


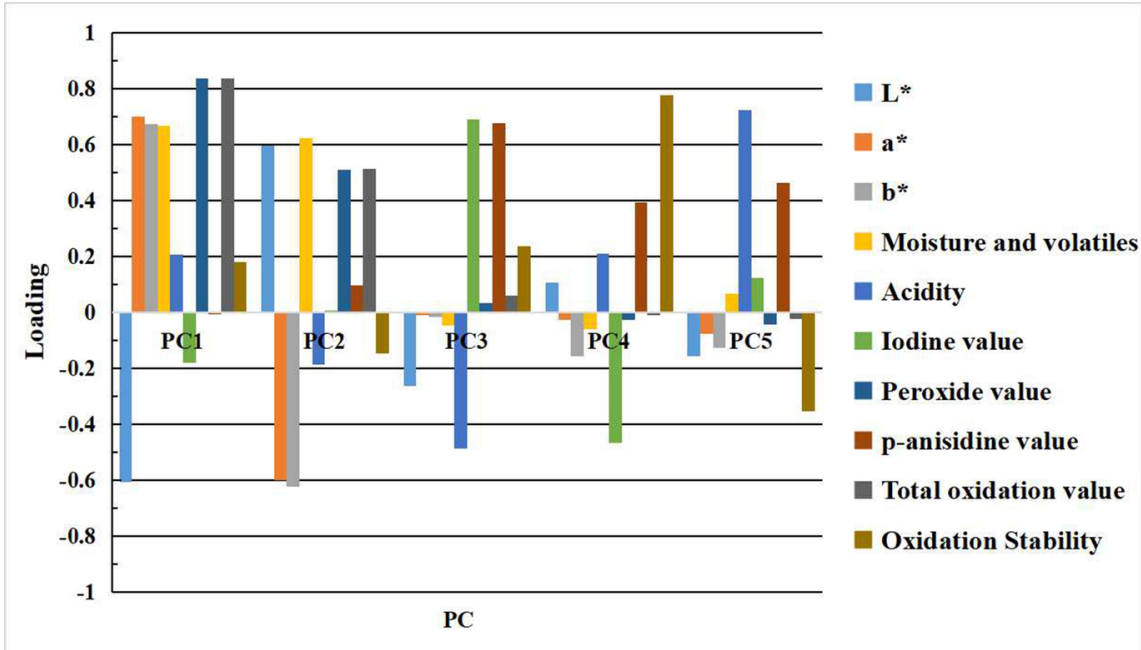


A

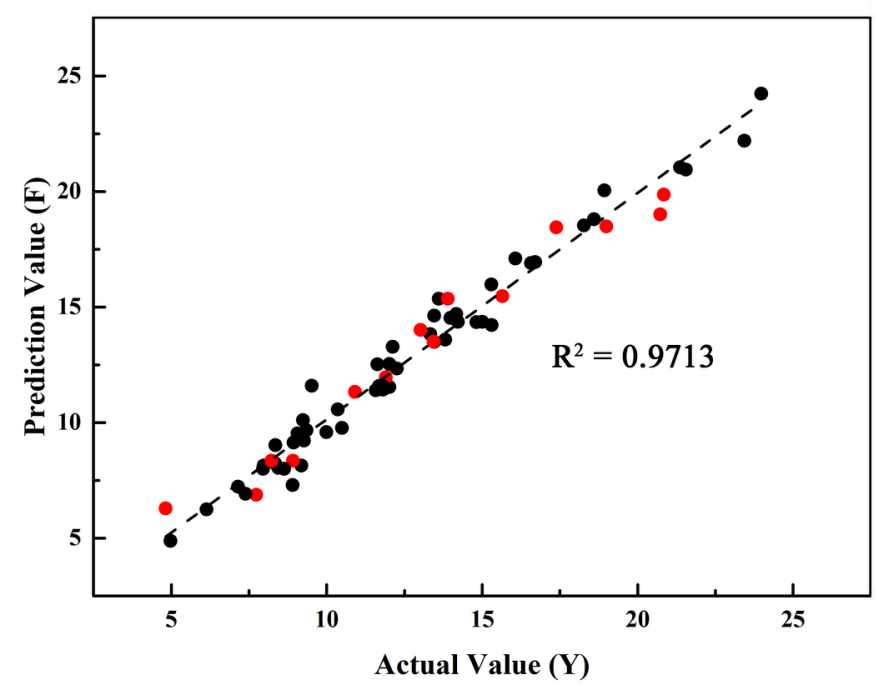

B

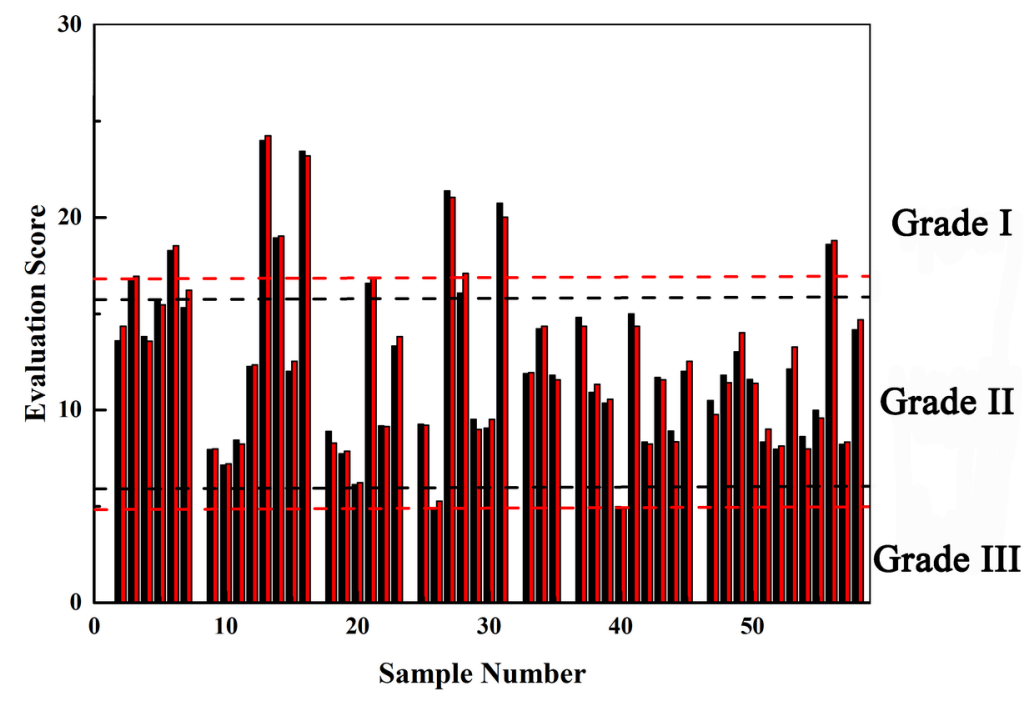

